\begin{tabular}{|c|l|}
\hline Title & Two-photon resonant excitation of a doubly excited state in He atoms by high-harmonic pulses \\
\hline Author(s) & Sekikawa, Taro; Okamoto, Tatsuya; Haraguchi, Eisuke; Y amashita, Mikio; Nakajima, Takashi \\
\hline Citation & $\begin{array}{l}\text { Optics Express, 16(26), 21922-21929 } \\
\text { https:/doi.org/_0.1364/0E.16.021922 }\end{array}$ \\
\hline Issue Date & 2008_12-22 \\
\hline Doc URL & http://hdl.handle.net/2115/45304 \\
\hline Rights & O2008 Optical Society of A merica \\
\hline Type & article \\
\hline File Information & OE16-26_21922-21929.pdf \\
\hline
\end{tabular}

Instructions for use 


\title{
Two-photon resonant excitation of a doubly excited state in He atoms by high-harmonic pulses
}

\author{
Taro Sekikawa, ${ }^{1 *}$ Tatsuya Okamoto, ${ }^{1}$ Eisuke Haraguchi, ${ }^{1}$ Mikio Yamashita, ${ }^{1}$ and \\ Takashi Nakajima ${ }^{2}$ \\ ${ }^{I}$ Department of Applied Physics, Hokkaido University, Kita 13 Nishi 8, Kita-ku, Sapporo 060-8628, Japan \\ ${ }^{2}$ Institute of Advanced Energy, Kyoto University, Gokasho, Uji, Kyoto 611-0011, Japan \\ corresponding author: sekikawa@eng.hokudai.ac.jp
}

\begin{abstract}
We experimentally demonstrate a two-color two-photon resonant excitation of the doubly excited $2 p^{2}{ }^{1} \mathrm{~S}$ state in helium atoms by the combination of 19th and 21st harmonic photons of a Ti:sapphire laser. Production of the $2 p^{2}{ }^{1} \mathrm{~S}$ state is confirmed by the experimental observation that the electron emission from this state does not depend on the direction of harmonic polarization. Our ab-initio theoretical results through the solution of time-dependent Schrödinger equation are consistent with the experimental results and confirm the first successful production of a doubly excited state by high-harmonic pulses.
\end{abstract}

(C)2008 Optical Society of America

OCIS codes: (190.4180) Multiphoton processes; (190.4160) Multiharmonic generation.

\section{References and links}

1. C. Spielmann, N. H. Burnett, S. Sartania, R. Koppitsch, M. Schnurer, C. Kan, M. Lenzner, P. Wobrauschek, and F. Krausz, "Generation of Coherent X-rays in the Water Window Using 5-Femtosecond Laser Pulses," Science 278, 661-664 (1997).

2. Z. Chang, A. Rundquist, H. Wang, M. M. Murnane, and H. C. Kapteyn, "Generation of Coherent Soft X rays at $2.7 \mathrm{~nm}$ Using High Harmonics," Phys. Rev. Lett. 79, 2967-2970 (1997).

3. J. Seres, E. Seres, A. J. Verhoef, G. Tempea, C. Streli, P. Wobrauschek, V. Yakovlev, A. Scrinzi, C. Spielmann, and F. Krausz, "Souce of coherent kiloelectronvolt x-rays," Nature 433, 596 (2005).

4. V. Ayvazyan, N. Baboi, J. Bähr, V. Balandin, B. Beutner, A. Brandt, I. Bohnet, A. Bolzmann, R. Brinkmann,

O. I. Brovko, J. P. Carneiro, S. Casalbuoni, M. Castellano, P. Castro, L. Catani, E. Chiadroni, S. Choroba, A.

Cianchi, H. Delsim-Hashemi, G. DiPirro, M. Dohlus, S. Düsterer, B. F. H. T. Edwards, A. A. Fateev, J. Feldhaus, K. Flöttmann, J. Frisch, L. Fröhlich, T. Garvey, U. Gensch, N. Golubeva, H. -J. Grabosch, B. Grigoryan, O Grimm, U. Hahn, J. H. Han, M. V. Hartrott, K. Honkavaara, M. Hüning, R. Ischebeck, E. Jaeschke, M. Jablonka1, R. Kammering, V. Katalev, B. Keitel, S. Khodyachykh, Y. Kim, V. Kocharyan, M. Körfer, M. Kollewe, D. Kostin, D. Krämer, M. Krassilnikov, G. Kube, L. Lilje, T. Limberg, D. Lipka, F. Lähl, M. Luong, C. Magne, J. Menzel, P. Michelato, V. Miltchev, M. Minty, W. D. Möller, L. Monaco, W. Müller, M. Nagl, O. Napoly, P. Nicolosi, D. Nölle, T. Nuñez, A. Oppelt, C. Pagani, R. Paparella, B. Petersen, B. Petrosyan, J. Pflüger, P. Piot, E. Plönjes, L. Poletto, D. Proch, D. Pugachov, K. Rehlich, D. Richter, S. Riemann, M. Ross, J. Rossbach, M. Sachwitz, E. L. Saldin, W. Sandner, H. Schlarb, B. Schmidt, M. Schmitz, P. Schmüser, J. R. Schneider, E. A. Schneidmiller, H.-J. Schreiber, S. Schreiber, A. V. Shabunov, D. Sertore, S. Setzer, S. Simrock, E. Sombrowski, L. Staykov, B. Steffen, F. Stephan, F. Stulle, K. P. Sytchev, H. Thom, K. Tiedtke, M. Tischer, R. Treusch, D. Trines, I. Tsakov, A. Vardanyan, R. Wanzenberg, T. Weiland, H. Weise, M. Wendt, I. Will, A. Winter, K. Wittenburg, M. V. Yurkov, I. Zagorodnov, P. Zambolin, and K. Zapfe, "First Operation of a Free-electron Laser Generating GW power radiation at 32 nm wavelength," Eur. Phys. J. D 37, 297-303 (2006).

5. Y. Kobayashi, T. Sekikawa, Y. Nabekawa, and S. Watanabe, "27-fs extreme ultraviolet pulse generation by high-order harmonics," Opt. Lett. 23, 64-66 (1998).

6. P. Tzallas, D. Charalambidis, N. A. Papadogiannis, K. Witte, and G. D. Tsakiris, "Direct observation of attosecond light bunching," Nature(London) 426, 267-271 (2003).

7. N. Miyamoto, M. Kamei, D. Yoshitomi, T. Kanai, T. Sekikawa, T. Nakajima, and S. Watanabe, "Observation of two-photon above-threshold ionization of rare gases by xuv harmonic photons," Phys. Rev. Lett. 93, 083903 (2004).

8. T. Nakajima and S. Watanabe, "Two-photon above-threshold-ionization cross sections of rare-gas atoms by xuv photons," Phys. Rev. A 70, 043412 (2004).

9. T. Sekikawa, A. Kosuge, T. Kanai, and S. Watanabe, "Nonliear optics in the extreme ultraviolet," Nature(London) 432, 605-608 (2004). 
10. Y. Nabekawa, H. Hasegawa, E. Takahashi, and K. Midorikawa, "Production of Doubly Charged Helium Ions by Two-Photon Absorption of an Intense Sub-10-fs Soft X-Ray Pulse at 42 eV Photon Energy," Phys. Rev. Lett. 94, 043001 (2005).

11. A. Kosuge, T. Sekikawa, X. Zhou, T. Kanai, S. Adachi, and S. Watanabe, "Frequency-Resolved Optical Gating of Isolated Attosecond Pulses in the Extreme Ultraviolet," Phys. Rev. Lett. 97, 263901 (2006).

12. Y. Nabekawa, T. Shimizu, T. Okino, K. Furusawa, H. Hasegawa, K. Yamanouchi, and K. Midorikawa, "Interferometric Autocorrelation of an Attosecond Pulse Train in the Single-Cycle Regime," Phys. Rev. Lett. 97, 153904 (2006).

13. M. Nagasono, E. Suljoti, A. Pietzsch, F. Hennies, M. Wellhöfer, J.-T. Hoeft, M. Martins, W. Wurth, R. Treusch, J. Feldhaus, J. R. Schneider, and A. Föhlisch, "Resonant two-photon absorption of extreme-ultraviolet free-electron-laser radiation in helium," Phys. Rev. A 75, 051406(R) (2007).

14. J. L. Krause, K. J. Schafer, and K. C. Kulander, "Higher-Order Harmonic Generation from Atoms and Ions in the High Intensity Regime," Phys. Rev. Lett. 68, 3535-3538 (1992).

15. Z. Chang, A. Rundquist, H. Wang, I. Christov, H. C. Kapteyn, and M. M. Murnane, "Temporal phase control of soft-X-ray harmonic emission," Phys. Rev. A 58, R30-R33 (1998).

16. J. W. Cooper, U. Fano, and F. Prats, "Classification of Two-Electron Excitation Levels of Helium," Phys. Rev. Lett. 10, 518-521 (1963).

17. R. P. Madden and K. Codling, "New Autoionizing Atomic Energy Levels in He, Ne, and Ar," Phys. Rev. Lett. 10, 516-518 (1963).

18. C. D. Lin, "Classification and supermultiplet structure of doubly excited states," Phys. Rev. A 29, 1019-1033 (1984).

19. M. Domke, K. Schulz, G. Remmers, G. Kaindl, and D. Wintgen, "High-resolution study of ${ }^{1} \mathrm{P}_{0}$ doubleexcitation states in helium," Phys. Rev. A 53, 1424-1438 (1996).

20. M. K. Odling-Smee, E. Sokell, P. Hammond, and M. A. MacDonald, "Radiative Decay of Doubly Excited States in Helium below the $\mathrm{He}^{+}(\mathrm{N}=2)$ Ionization Threshold," Phys. Rev. Lett. 84, 2598-2601 (2000).

21. F. Penent, P. Lablanquie, R. I. Hall, M. Žitnik, K. Bučar, S. Stranges, R. Richter, M. Alagia, P. Hammond, and J. G. Lambourne, "Observation of Triplet Doubly Excited States in Single Photon Excitaion from Ground State Helium," Phys. Rev. Lett. 86, 2758-2761 (2001).

22. X. M. Tong and C. D. Lin, "Double photoexcitation of He atoms by attosecond xuv pulses in the presence of intense few-cycle infrared lasers," Phys. Rev. A 71, 033406 (2005).

23. B. A. deHarak, J. G. Childers, and N. L. S. Martin, "Ejected electron spectrum of He below the N=2 threshold," Phys. Rev. A 74, 032714 (2006).

24. T. Morishita, S. Watanabe, and C. D. Lin, "Attosecond Light Pulses for Probing Two-Electron Dynamics of Helium in the Time Domain," Phys. Rev. Lett. 98, 083003 (2007).

25. P. Salieres, A. L'Huillier, and M. Lewenstein, "Coherence Control of High-Order Harmonics," Phys. Rev. Lett. 74, 3776-3779 (1995).

26. X. Tang, H. Rudolph, and P. Lambropoulos, "Nonperturbative time-dependent theory of helium in a strong laser field," Phys. Rev. A 44, R6994 (1991).

27. T. Nakajima, and G. Buica, "Above-threshold ionization of Mg by linearly and circularly polarized laser fields: Origin of the subpeaks in the photoelectron energy spectra," Phys. Rev. A 74, 023411 (2006).

28. T. Nakajima, "Above-threshold ionization by chirped laser pulses," Phys. Rev. A 75, 053409 (2007).

\section{Introduction}

Development of ultrashort pulse lasers has led to the generation of coherent extremeultraviolet (XUV) and soft x-ray (SXR) light [1,2]. Synchrotron orbital radiation (SOR) has been a unique light source in XUV and SXR spectroscopy for a long time and providing light with excellent spatial coherence. Indeed study on the relaxation and dissociation dynamics of atoms and molecules using a one-photon transition by SOR has been one of the main research issues in XUV linear spectroscopy. Although the usefulness of XUV nonlinear spectroscopy is rather obvious, to go beyond the linear region is not possible with SOR, since the available peak intensity is very limited due to the lack of temporal coherence. Alternative light sources in the XUV or SXR region are based on lasers: The highest photon energy of the high harmonics generated by ultrashort pulse lasers was extended to $1 \mathrm{keV}$ and free-electron lasers (FELs) for the XUV region have been developed [3,4]. Yet, the available intensity is not sufficient, and as a result, experimental investigation of nonlinear optics in the XUV or SXR region has been a rather uncharted territory so far.

Further development of high harmonics by femtosecond lasers has paved the way for XUV nonlinear optics in the last few years [5-10]: Two-photon ionization of helium atoms was demonstrated using the 9th harmonic of a Ti:sapphire (TiS) laser and applied to the autocorrelation measurements [5]. Moreover, above-threshold ionization (ATI) was also observed and attosecond pulses were characterized using the ATI process $[9,11,12]$. Note 
that all these experiments are based on the non-resonant processes in that neither bound nor autoionizing states are resonantly involved during the ionization processes. If we are to use XUV pulses from the high harmonics for ultrafast nonlinear XUV spectroscopy, a two-photon resonant excitation to the final state of interest is greatly desired, since highly excited states of atoms and molecules, which have tens of eV state energy and cannot be reached by the onephoton process from the initial state due to the parity selection rule, will be within reach by the two-photon process.

Although the two-photon excitation to a doubly excited state with a FEL was suggested recently $[4,13]$, it was difficult to selectively excite a specific state, since the photon energy of the FEL was fixed to $38.5 \mathrm{eV}$. In contrast, high harmonics have multiples of the photon energy of the fundamental laser up to the cut-off energy [14], and not only one of the harmonics (one-color two-photon excitation) but a combination of the two (two-color twophoton excitation) may be used to resonantly excite the final state of interest. Moreover, the center energy of each harmonic can be shifted by changing the laser chirp. That is, use of the negatively and positively chirped laser pulses enables us to almost continuously shift (tune) the center photon energy upwards and downwards, respectively [15]. Because of this practical tunability of the photon energy, high harmonics are very useful for XUV and SXR nonlinear spectroscopy of atoms and molecules, etc. Toward this goal, experimental observation of twophoton resonant excitation of a doubly excited state by high-harmonic pulses would serve as a cornerstone for ultrafast XUV nonlinear spectroscopy.

In this paper, we report the first experimental observation of two-photon resonant excitation to the doubly excited state of helium atoms by XUV harmonic photons together with our ab-initio theoretical results. Here, we focus on the helium atom as a nonlinear optical medium, since it is the simplest three-body system consisting of an atomic nucleus and two electrons, which can be rigorously treated in the theoretical description. One of the interesting properties of helium atoms is the presence of doubly excited states [16-24, 13] All the doubly excited states of helium atoms are located far above the ionization threshold, and hence they autoionize. Although the doubly excited states of helium were first observed more than forty years ago using SOR [17], they are still under extensive investigation because helium is a proto-type system with electron-electron correlations. Related to our work, optical excitation of the doubly excited states by XUV attosecond pulses $[22,24]$ has been theoretically studied recently.

Figure 1(a) shows the energy diagram of the relevant states of helium atom. In this paper we focus on the $2 p^{2}{ }^{1} \mathrm{~S}$ state, because the excitation energy is $62.1 \mathrm{eV}$ and corresponds to the summed energy of the 19 th and 21 st harmonics. The $2 p^{2}{ }^{1} \mathrm{~S}$ state is known to decay into a $1 s \varepsilon s$ continuum state through the autoionization with a lifetime of $140 \mathrm{fs}$ [22], where the notation
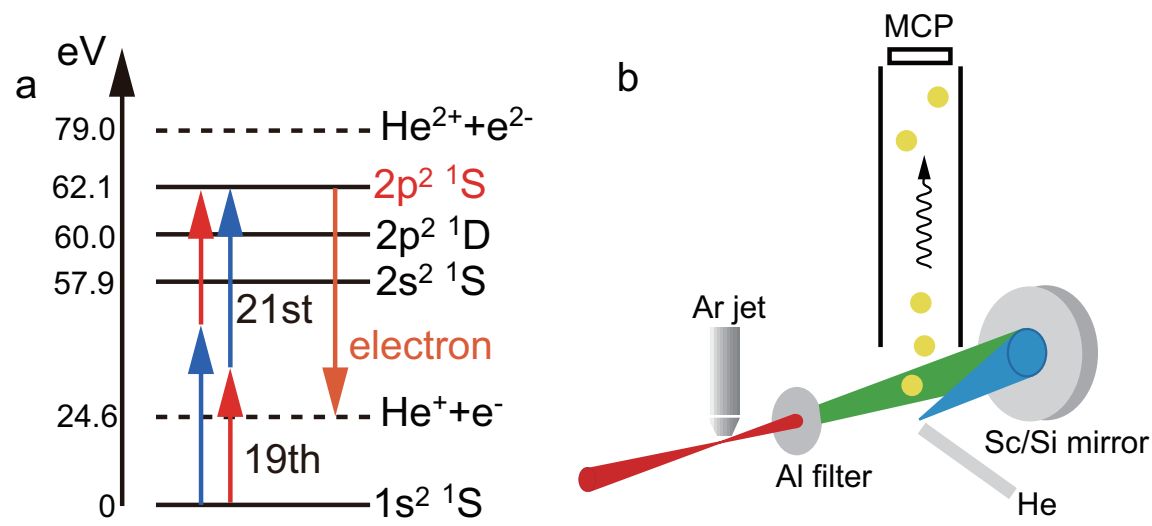

Fig. 1. (a) Schematic energy diagram of helium atoms. One electron in the $2 p^{2}{ }^{1} \mathrm{~S}$ state is ejected into the vacuum state (autoionizing electron) with a kinetic energy of $37.5 \mathrm{eV}$ and the other relaxes to the $1 s$ orbital. (b) Experimental setup to detect a doubly excited state in helium. 
" $\varepsilon s$ " represents the electron in the $s$ continuum with a kinetic energy of $\varepsilon(>0)$. Therefore, if we are able to resonantly excite the $2 p^{2}{ }^{1} \mathrm{~S}$ state by two-photon (19th and 21 st harmonic) absorption, we should be able to confirm the process by detecting autoionizing electrons in the $s$ wave at a kinetic energy of $37.4 \mathrm{eV}$. In order to confirm the successful detection of autoionizing $\varepsilon s$ electrons, we have measured the electron signals with two different polarization geometries, the detail of which will be explained later in this paper.

\section{Experimental}

The experimental setup for the two-photon excitation shown in Fig. 1b was as follows: 800- $\mu \mathrm{J}$, 30 -fs TiS laser pulses at a repetition rate of $1 \mathrm{kHz}$ were focused on a pulsed jet of argon gas; the gas nozzle was positioned after the laser focus so that we can attain a good phase matching for high harmonic generation [25]. The peak intensity for high-harmonic generation was $2.5 \times$ $10^{14} \mathrm{~W} / \mathrm{cm}^{2}$. The photon energies of high harmonics were adjusted by changing the amount and sign of the chirp through the pulse compressor of the TiS laser system. The 19th and 21st harmonics were separated from the fundamental laser by an Al filter and then focused into helium atoms by a concave $\mathrm{Sc} / \mathrm{Si}$ multilayer mirror with a focal length of $5 \mathrm{~cm}$. The center wavelength of this mirror was $42 \mathrm{~nm}$ with a 4-nm bandwidth. The kinetic energy of the ejected electrons was analyzed by a time-of-flight-type electron spectrometer with a magneticbottle to have a wide detection angle. We have applied an electric field to the flight tube to slow down the fast electrons, which results in the improvement of energy resolution. From the temporal widths of the electronic trigger pulses, the energy resolution of the detector around $37 \mathrm{eV}$ was estimated to be $420 \mathrm{meV}$. As we have already mentioned and will explain more in detail later in this paper, light polarization is a very important parameter to confirm the resonant excitation to the doubly excited $2 p^{2}{ }^{1} \mathrm{~S}$ state. Therefore, the direction of linear polarization of the high-harmonic pulses was rotated by the rotation of linear polarization of the fundamental laser beam with a half-wavelength plate. Since the $\varepsilon p$ electrons produced by one-photon ionization with high harmonics obey the $\cos ^{2} \theta$ distribution law where $\theta$ is a polar angle between the polarization direction of the harmonics and the detection direction, we can estimate the collection angle of the electron detector by measuring the $\varepsilon p$ electrons for the two

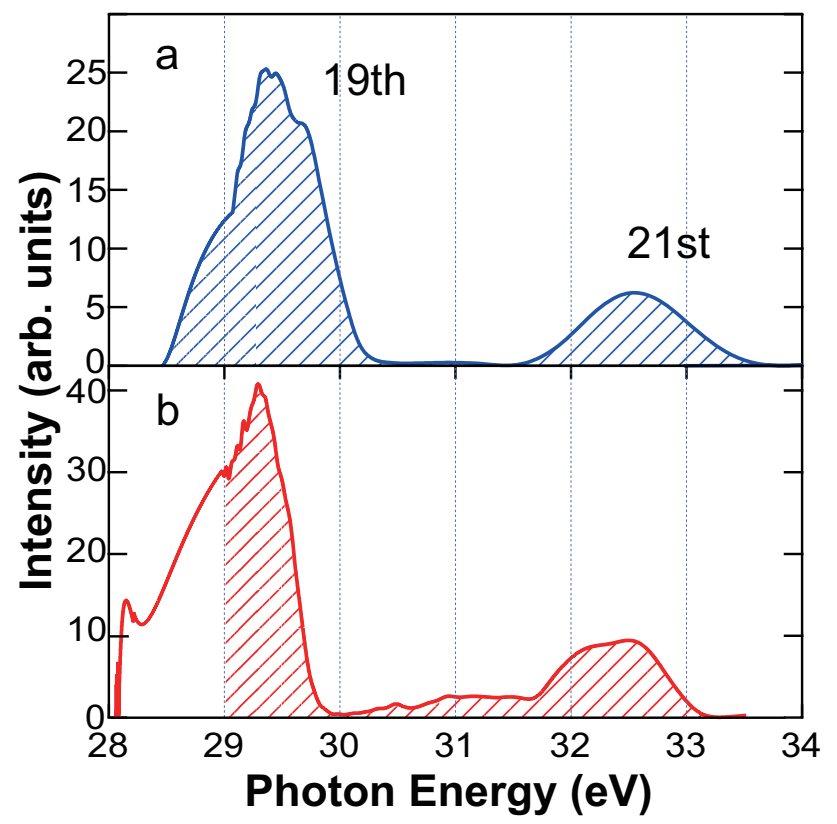

Fig. 2. Spectra of the 19th and 21st harmonics of a TiS laser pulse with different center photon energies. The hatched areas indicate the spectral components that contribute to the two-photon excitation to the $2 p^{2}{ }^{1} \mathrm{~S}$ state.

\#103985 - \$15.00 USD Received 11 Nov 2008; revised 3 Dec 2008; accepted 7 Dec 2008; published 17 Dec 2008 (C) 2008 OSA 22 December 2008 / Vol. 16, No. 26 / OPTICS EXPRESS 21925 
polarization geometries, i.e., parallel and perpendicular linear polarization with respect to the detection direction. The intensity ratio of the $\varepsilon p$ electrons signal for the parallel and perpendicular linear polarization geometries was found to be $3.0 \pm 0.1$, from which we estimated the collection polar angle as about 58 degrees. We accumulated the electron signal for 4 hours for each spectrum.

Figure 2 shows the spectra of the 19th and 21st harmonics used for the experiment. The hatched areas in the spectra indicate the spectral components which could, according to the law of energy conservation, presumably contribute to the resonant excitation of the $2 p^{2}{ }^{1} \mathrm{~S}$ state. Since the center energies of the spectra in Fig. 2(b) are slightly shifted to the lower energy than those in Fig. 2a, only 60\% energy of the 19th harmonic is expected to participate in the two-photon excitation for the case of Fig. 2(b).

\section{Observation of the doubly excited state}

Using these high-harmonic pulses, the electron spectra of helium for the kinetic energy between 28 and $42 \mathrm{eV}$ were measured and are shown in Figs. 3a and 3b with error bars which correspond to the harmonic spectra in Fig. $2 \mathrm{a}$ and $2 \mathrm{~b}$, respectively. In Figs. $3 \mathrm{a}$ and $3 \mathrm{~b}$ the solid and broken lines represent the results for the parallel and perpendicular linear polarization geometries, respectively. Although the 37th to 41st harmonics are far out of the designed reflectance bandwidth of the $\mathrm{Sc} / \mathrm{Si}$ mirror and must be already extremely weak at the target, the huge peaks by those harmonics appear in the spectra, since they originate from the onephoton processes. Nevertheless, the signal with a kinetic energy of about $37 \mathrm{eV}$, shown by the

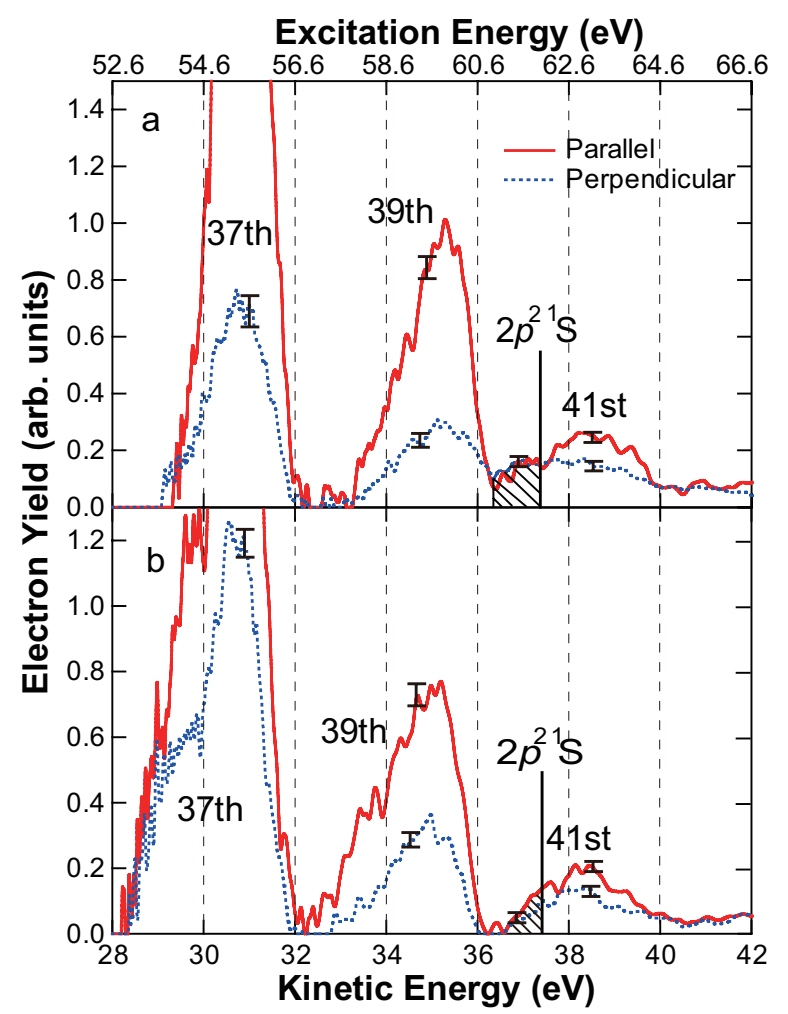

Fig. 3. (a) and (b) Electron spectra of helium atoms excited by the 19th and 21st harmonics with spectra of Fig $2 a$ and $2 b$, respectively. The solid and broken lines with error bars indicate the electron spectra observed in the directions parallel and perpendicular to the light polarization, respectively. The vertical solid lines indicate the expected center kinetic energies of the autoionizing electrons from the $2 p^{2}{ }^{1} \mathrm{~S}$ state. The hatched area corresponds to the autoionizing electrons ejected from the $2 p^{2}{ }^{1} \mathrm{~S}$ state together with the 37th, 39th, and 41st harmonics.

\#103985 - \$15.00 USD Received 11 Nov 2008; revised 3 Dec 2008; accepted 7 Dec 2008; published 17 Dec 2008 (C) 2008 OSA 
hatched area in Fig. 3(a), seems to originate from the two-photon resonant excitation of doubly excited $2 p^{2}{ }^{1} \mathrm{~S}$ state, which must be confirmed. Here, the hatched area cannot come from the two-photon processes involving one harmonic (39th or 41st) photon and one fundamental photon. If that were the case, sidebands would have been appeared by the huge one-photon ionization peaks.

We have confirmed the excitation of the $2 p^{2}{ }^{1} \mathrm{~S}$ state by two completely different experimental methods as we explain below: In the first confirmation method, we utilized the two different harmonic spectra shown in Figs. 2(a) and 2(b), and checked the change, if any, of the electron spectra shown in Figs. 3(a) and 3(b). As we have already mentioned, the excitation efficiency of the $2 p^{2}{ }^{1} \mathrm{~S}$ should become smaller when we employ the harmonics with the spectra shown in Fig. $2 \mathrm{~b}$, because the spectral intensity that can participate to excite the $2 p^{2}{ }^{1} \mathrm{~S}$ state was reduced to $60 \%$. Consistent with this argument, the number of electrons with a kinetic energy of about $37 \mathrm{eV}$ indeed decreased, as shown in Fig. 3(b). It is difficult, however, to quantitatively estimate the decrease because of the overlap of the relevant twophoton signal with the one-photon signal by the 39th and 41st harmonics. In the second confirmation method, we made use of the angle-dependence of the ejected electrons by the one- and two-photon processes. Since the $2 p^{2}{ }^{1} \mathrm{~S}$ state necessarily decays into the 1 ses continuum state through autoionization, the ejected electrons are the $\varepsilon s$ electrons with an isotropic spatial distribution upon ejection. This means that the two-photon resonant signal has no polarization dependence, which is indeed what we find in Fig. 3. In contrast, onephoton ionization results in the ejection of $\varepsilon p$ electrons which now obeys the $\cos ^{2} \theta$ distribution law and hence exhibits polarization dependence for our finite detection angle (about 58 degrees as estimated before). This clearly shows that we have successfully demonstrated the two-photon resonant excitation of the doubly excited $2 p^{2}{ }^{1} \mathrm{~S}$ state by harmonic photons.

\section{Comparison with ab-initio theory}

For further confirmation, we have also undertaken ab-initio theoretical calculations by solving the time-dependent Schrödinger equation (TDSE) for the helium atom with two active electrons [26, 27]. Briefly, the computational procedure can be summarized as follows: First, we construct the two-electron states of helium by diagonalizing the two-electron Hamiltonian with more than 7000 configurations for the S, P, and D symmetries in a spherical box of 1000 atomic units. Then, we expand the time-dependent two-electron wavefunction under the harmonic field as a superposition of two-electron states multiplied by time-dependent expansion coefficients. Thus, the TDSE is reduced to a set of first-order differential equations for the time-dependent expansion coefficients. The number of first-order differential equations we solve is 8000. As for the field, the harmonic field is assumed to contain two main components corresponding to the 19th and 21 st harmonics, and three subsidiary components corresponding to the 37 th, 39th, and 41 st harmonics. However, as our experimental results in Figs. 2 and 3 indicate, photon energies of the subsidiary harmonics seem to have shifted since these harmonics are produced in the argon gas under the presence of lots of electrons due to ionization. To phenomenologically account for the shift, we have shifted the photon energies for the three subsidiary harmonic components. Thus the harmonic photon energies employed in the calculations are $29.45 \mathrm{eV}$ and $32.5 \mathrm{eV}$ for the main harmonics, and $55.48 \mathrm{eV}, 59.31 \mathrm{eV}$, and $63.23 \mathrm{eV}$ for the subsidiary harmonics, respectively. It is also very likely that the harmonics are negatively chirped, since they are produced under the presence of lots of electrons. To account for the possible chirp, the harmonic pulse is assumed to have a cosinesquared temporal envelope function with a $4 \mathrm{fs}$ (FWHM) duration with a linear negative chirp [28], which has a spectral bandwidth of the transform-limited $2 \mathrm{fs}$ pulse. The peak intensities employed in the calculations are $3.8 \times 10^{9} \mathrm{~W} / \mathrm{cm}^{2}$ and $1.2 \times 10^{9} \mathrm{~W} / \mathrm{cm}^{2}$, respectively, for the main (19th and 21st) harmonics, which were estimated from the experimentally measured pulse energies and the beam diameters. Because the pulse energies of the three subsidiary harmonics were too weak to measure, we temporarily assumed that they are approximately

\#103985 - \$15.00 USD Received 11 Nov 2008; revised 3 Dec 2008; accepted 7 Dec 2008; published 17 Dec 2008 
$<10^{-6}$ of the 19th harmonic. In order to obtain the electron spectra comparable to the experimental ones, we have slightly scaled the one-photon peaks for each subsidiary harmonic component.

The solid and broken lines in Fig. 4(a) represent the calculated electron spectra associated with the two-photon absorption followed by the electron ejection into the $\varepsilon s$ and $\varepsilon d$ waves, respectively. For clarity, in Fig. 4a, we have omitted the electron signals originating from the one-photon processes by the subsidiary harmonics. Some of the distinct resonant peaks, such as $2 s^{2}{ }^{1} \mathrm{~S}$ at $57.84 \mathrm{eV}, 2 p^{2}{ }^{1} \mathrm{D}$ at $59.90 \mathrm{eV}, 2 p^{2}{ }^{1} \mathrm{~S}$ at $62.10 \mathrm{eV}$, and $2 s 3 s{ }^{1} \mathrm{~S}$ at $62.96 \mathrm{eV}$, are labeled in Fig. 4(a). To be more realistic, we now include the electron signals originating from the one-photon processes by the subsidiary harmonics, and applied a window function with a $420 \mathrm{meV}$ width of the Gaussian function. After taking into account the anisotropic and isotropic spatial distribution of the electron ejection by the one- and two-photon ionization, respectively, the calculated results are shown in Fig. 4(b) for the parallel and perpendicular linear geometries. Because of the huge peaks induced by the 37th, 39th, and 41st subsidiary harmonics, some of the peaks associated with the two-photon processes shown in Fig. 4a are buried in Fig. 4b. Nevertheless, the peak associated with the resonant two-photon excitation of the doubly excited $2 p^{2}{ }^{1} \mathrm{~S}$ state can be clearly seen in Fig. 4(b). This theoretical finding is quite consistent with the experimental results in Fig. 3(a), and again we have confirmed that the $2 p^{2}$ ${ }^{1} \mathrm{~S}$ state has been successfully excited through the two-photon resonant processes.

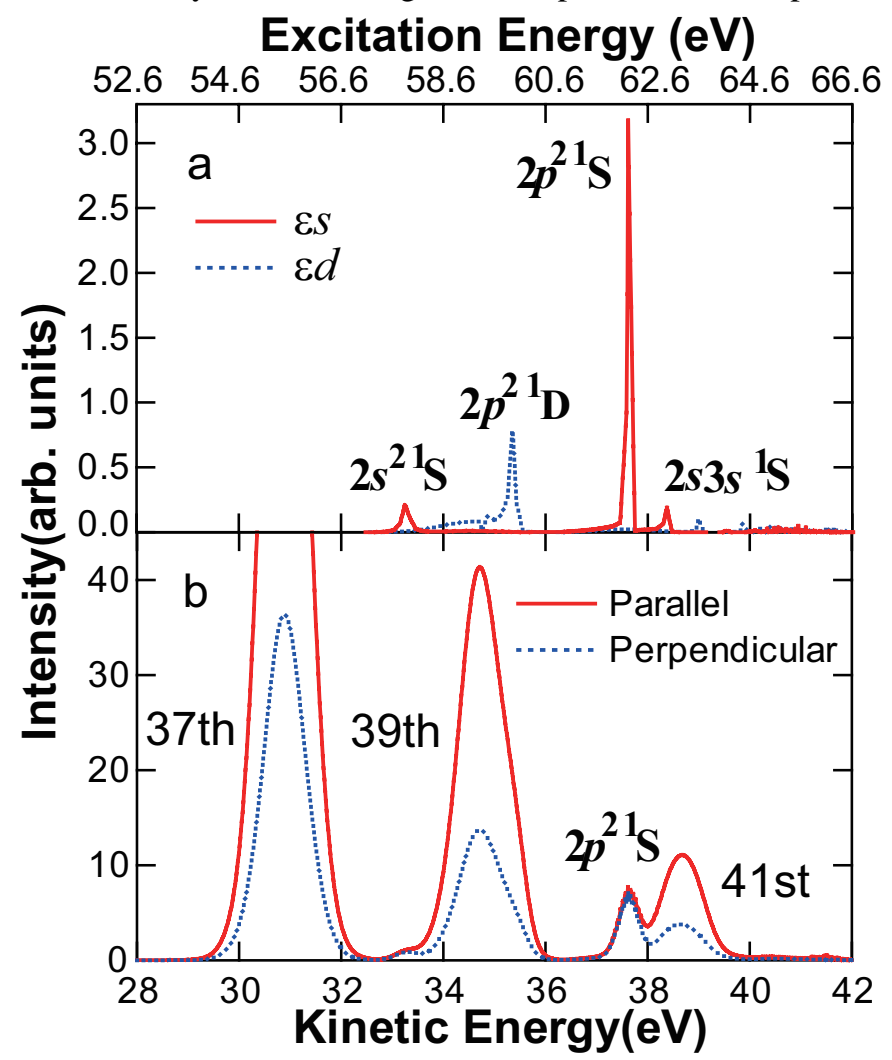

Fig. 4. (a) Calculated electron spectra through the two-photon excitation by the two main (19th and 21st) harmonics. The solid and broken lines represent the electron signal into the $\varepsilon s$ and $\varepsilon d$ waves, respectively. (b) Calculated electron spectra by the two main harmonics and three subsidiary (37th, 39th, and 41st) harmonics under the parallel (solid line) and perpendicular (broken line) linear polarization geometries. In Fig. 4b, detection angle and the energy resolution of the electron spectrometer have been taken into account. 


\section{Summary}

In summary, we have experimentally demonstrated the two-color two-photon resonant excitation of the doubly excited $2 p^{2}{ }^{1} \mathrm{~S}$ state in helium by the combination of the 19th and 21st harmonics from the TiS laser. We have also performed the ab-inito theoretical calculations and found a good agreement with the experimental results. Our results can serve as a doorway toward ultrafast XUV nonlinear spectroscopy in that highly excited states of atoms and molecules, which have never been accessed by the one-photon process via SOR due to the parity selection rule, will become within reach by the two-photon process.

\section{Acknowledgments}

This work is partially supported by Japan Society of Promotion of Science, Japan Science and Technology Agency, Murata Science Foundation, and MATSUO FOUNDATION. The work by T.N. was supported by Research Foundation for Opto-Science and Technology. 